\title{
Spatial variability of the major chemistry of the Antarctic ice sheet
}

\author{
R. Mulvaney and E. W. Wolr \\ British Antarctic Survey, Natural Environment Research Council, Cambridge CB3 OET, England
}

\begin{abstract}
A compilation of reliable data for sodium, nitrate, chloride and sulphate has been made. $\mathrm{NO}_{3}$ concentrations are remarkably consistent across Antarctica, though there appears to be some correlation with altitude and accumulation rate. Post-depositional loss of $\mathrm{NO}_{3}{ }^{-}$is important at low-accumulation sites. Cl concentration (either measured directly or calculated from $\mathrm{Na}^{+}$via the seasalt ratio) decreases with distance from the coast, though the decline is less rapid if the coastal topography is not steep or mountainous. Excess sulphate (xs $\mathrm{SO}_{4}^{2}$ ) concentration (herc calculated from normal sea-salt ratios with $\mathrm{Na}^{+}$or $\mathrm{Cl}$ ) also declines with distance from the coast, though less quickly than $\mathrm{Cl}^{-}$. Fractionation of sea-salt ions makes the calculation of $\mathrm{xs} \mathrm{SO}_{4}{ }^{2-}$ unccrtain.
\end{abstract}

\section{INTRODUGTION}

Over the last three decades, ice cores have been drilled at numerous locations in Antarctica. They have ranged from cores of $10 \mathrm{~m}$ or less to the decpest core at Vostok, which is now $2546 \mathrm{~m}$ long and spans 220000 years. Soluble ionic chemistry has been measured in many of these cores, and temporal variations have been interpreted in terms of changing atmospheric sources and transport. However, both sources and transport arc poorly understood even under current conditions, as is the air snow transfer function, and this hinders temporal interpretations.

One approach to understanding thcse factors better is to investigate how chemical concentrations vary spatially and seasonally within Antarctic snowfall. Ideally, this would be done with year-round surveys of atmospheric chemistry at many sites. However, aerosol sampling has been conducted at only a very few sites, often for only part of the year, and sometimes too close to manned stations, so that the results are not representative of the regional background. Exceptions to this are the cxcellcnt acrosol records from Neumayer (Wagenbach and others, 1988) and Mawson (Savoie and others, 1992).

The spatial distribution of chemistry in the snowpack can be used as a surrogate for the atmosphere. The ITASE (International Trans-Antarctic Scientific Expedition) sample collections planned for the next lew years will provide an excellent data network for doing this. However, much data already exist. It is likely that closer study of them may highlight more clearly some of the questions that ITASE activities need to pursue. Compilations have been made previously for some parameters such as accumulation rate (Giovinetto and Bull, 1987; Giovinetto and others, 1990 and oxygen-isotopic ratios (Morgan, 1982). In this paper, we compile for the first time all the existing and reliable data for soluble chemistry and provide an interpretation of their spatial variability.

\section{CHEMICAL SPECIES}

The major soluble impurities in Antarctic ice are:

(1) Sea-salt ions (part of $\mathrm{Na}^{+}, \mathrm{Mg}^{2+}, \mathrm{K}^{+}, \mathrm{Ca}^{2+}, \mathrm{Cl}^{-}$, $\mathrm{SO}_{4}{ }^{2-}$ ).

(2) $\mathrm{H}_{2} \mathrm{SO}_{4}$, derived from oxidation of marine biogenic emissions of DMS and from volcanism (both background and sporadic events).

(3) $\mathrm{HNO}_{3}$, probably derived from the stratosphere and from tropical lightning (Legrand and Kirchner, 1990). At this time, significant anthropogenic contributions are not discernible in Antarctic snow (away from stations) for either sulphate or nitrate.

(4) $\mathrm{H}_{2} \mathrm{O}_{2}$, a product of photochemical processes in the atmosphere.

(5) T'errestrial input, represented by elements such as $\mathrm{Al}$ and $\mathrm{Ca}$, as well as by insoluble dust.

More minor components that have been measured include methanesulphonic acid (MSA) (Legrand and others, 1992), $\mathrm{NH}_{4}{ }^{+}$, organic acids (Legrand and Saigne, 1988) and other trace metals (Görlach and Boutron, 1992; Suttie and Wolff, 1992).

The minor components have been measured only at a lew sites and a spatial survey is not yet appropriate. One of the more interesting species may be MSA, because it has only one known source foxidation of biogenically produced DMS). Current knowledge of MSA has been summarized by Legrand and others (1992). $\mathrm{H}_{2} \mathrm{O}_{2}$ has also been measured at only a few sites and interest in it continues to grow. The terrestrial elements have been reported in a number of ways (particle numbers, dust concentrations, $\mathrm{Al}$ and $\mathrm{Ca}$ ) and it is difficult to produce a widespread or coherent spatial survey, although this would be valuable. In this paper, we therefore concen- 
trate on the first three categories of soluble species.

I.arge amounts of data exist for $\mathrm{Cl}^{-}, \mathrm{NO}_{3}{ }^{-}$and $\mathrm{SO}_{4}{ }^{2}$ concentrations across Antarctica. Wc can supplement the $\mathrm{Cl}$ data at other sites where $\mathrm{Na}^{+}$has been measured by converting to $\mathrm{Cl}^{-}$using a sea-salt ratio. This procedurc ignores the documented cases where $\mathrm{Na}^{+}$and $\mathrm{Cl}$ are not in sea-salt ratio but, generally, the discrepancy for these two elements is at most a few per cent. Since we do not attempt to look at the fractionation of other sea-salt elements, which requires detailed study of data from single sites or single snowfalls, we have not used $\mathrm{Mg}^{2+}$, $\mathrm{K}^{+}$or $\mathrm{Ca}^{2+}$ data.

In summary, this paper will compilc data for the first three components of the soluble ionic components, using $\mathrm{Cl}^{-}$(and $\mathrm{Na}{ }^{-}$), $\mathrm{NO}_{3}$ and $\mathrm{SO}_{4}{ }^{2-}$ data. For $\mathrm{SO}_{4}{ }^{2-}$, we calculatc xs $\mathrm{SO}_{4}{ }^{2}$ from normal sca-salt $\mathrm{SO}_{4}{ }^{2} / \mathrm{Cl}$ or $\mathrm{SO}_{4}^{2} / \mathrm{Na}^{+}$ratios, i.e.:

$$
\begin{aligned}
\mathrm{xSSO}_{4}{ }^{2} & =\mathrm{SO}_{4}{ }^{2-}-0.103 \mathrm{Cl}^{-} \text {or } \\
\mathrm{xs} \mathrm{SO}_{4}{ }^{2} & =\mathrm{SO}_{4}{ }^{2} \quad 0.120 \mathrm{Na}^{+} \\
& \begin{array}{r}
\text { where all species are in } \\
\mu \mathrm{l}^{1} .
\end{array}
\end{aligned}
$$

This means that xs $\mathrm{SO}_{4}{ }^{2-}$ here is in effect the sum of two factors: (i) acidic sulphate and (ii) the deficit (or excess) of sulphate in sea-salt aerosol. The latter factor may be significant at near-coastal sites. Minikin and others (1994) have pointed out that negative $\mathrm{xs} \mathrm{SO}_{4}{ }^{2}$ values are often found at coastal sites, implying that a different ratio is appropriate for marine acrosol, at lcast for somc pcriods of the year. Therefore, they calculated xs $\mathrm{SO}_{4}{ }^{2}$ using lower ratios. Because we cannot say what ratio might be appropriate for cach site, we use the normal sea-salt ratio to quote xs $\mathrm{SO}_{4}{ }^{2-}$ for all sites, except for those of Minnikin and others where we have used their xs $\mathrm{SO}_{4}{ }^{2-}$ values. In Table 1 , we have also shown the effect of the conventional calculation using normal sea-salt ratios on their values.

\section{CRITERIA FOR INCLUDING DATA}

For data to be included in this compilation, they must be of good quality and representative of the chemistry at the site.

Good-quality data require careful, accuratc analysis and non-contaminating sample collection. In recent years, scientists have become more aware of the need to collect samples in clean bottles without adding contamination. The introduction of ion chromatography has made analysis of ions particularly anions) much easier and analyses should, in careful hands, be accuratc to $5 \%$ or so. We have accepted data where ion chromatography (or, for $\mathrm{Na}^{\dagger}$, atomic-absorption or equivalent methods) has becn used, and where suitable sample-collection techniques are believed to have been used. Where there are no other data for a site, we have accepted (mainly older) data not cxplicitly mecting these critcria but caution that the accuracy of some of these data is uncertain.

It is very important to use data that represcnt the site in question. For that reason, we have attempted to exclude data from any sporadic events (such as volcanic cruptions) where they significantly affect the mean concentration. Since no significant pollution trend has so far been observed in Antarctic snow for the species wc have compiled, we do not need a totally common timescale between sites to achieve comparability, but have gencrally uscd data from short cores covering only recent decades. Since there can be large scasonal variations, data should only be accepted where they cover complete years or average many ycars. However, we have not excluded the French D-sites, where many of the samples cover only 3 years (Legrand and Delmas, 1985). Additionally, at least for nitratc, there is mounting evidence that post-depositional changes occur in concentrations in the surface layers (Neubauer and Heumann, 1988; Mayew-ski and Legrand, 1990; Wolff, in press). For both these last two reasons, data from collections of only near-surface snow cannot be used to infer a background annual mean and cannot be comparcd to data from other sites. 'This is unfortunate, as such data are available from largc areas of the continent where no other data exist (Kamiyama and others, 1989; Dahc and others, 1992) but we have had to exclude them. Because of the near-surfacc loss of nitrate cvident particularly at low-accumulation sites such as Vostok, data from below $1 \mathrm{~m}$ depth are used where possible but this is discussed later.

\section{DATA AND DISGUSSION}

The data accepted by the above criteria are listed in Table 1 and illustrated in Figures 1-3. The major factors likely to influence concentrations are the distance from the coast, altitude and snow-accumulation rate (listed in Table 1). Sccondary factors such as temperature, sunlight received, typical wind speeds and seasonality of deposition may also strongly affect the average concentrations. A problem with analysing the data according to these variables is that many of them vary together. For example, across most of Antarctica, as distance from the coast increases, altitude also increases while temperature and accumulation rate decrease. We discuss each of $\mathrm{Cl}^{-}$, $\mathrm{NO}_{3}{ }^{-}$and $\mathrm{SO}_{4}{ }^{2-}$ in the light of these factors.

\section{1. $\mathrm{Cl}^{-}$}

$\mathrm{Cl}^{-}$(here sometimes measured directly but at some sites calculated from $\mathrm{Na}^{+}$) is derived mainly from sea spray. Even where $\mathrm{HCl}$ is apparently present, it is mainly derived from the reaction of $\mathrm{NaCl}$ and $\mathrm{H}_{2} \mathrm{SO}_{4}$ (Legrand and Delmas, 1988a). We therefore expect that distance from the sea and, to some extcnt, altitude, will be crucial factors. We calculate distance ignoring sea ice, because this is highly variable and scasonal, and there are normally shore leads providing at least some open water at the coast. In Figure 4 we present $\mathrm{Cl}^{-}$data from three data sets: the French chain of D sites, the Ross Icc Shelf and the Filchner-Ronne Ice Shelf (FRIS), including Berkner Island and the nearby Coats Land region. Herron (1982) has previously calculated an exponcntial relationship for $\mathrm{Cl}^{-}$against altitude for the Ross Ice Shelf, while Minikin and others (1994) have calculated an exponential relationship against distance for the FRIS. As cxplained carlier, altitude generally increases with distance from the coast in Antarctica, so either approach may be valid, depending on the topography. For ice 
Table 1. Data used in this analysis

\begin{tabular}{|c|c|c|c|c|c|c|c|c|c|c|}
\hline Site & lat. & Long. & Altitude & $\begin{array}{l}\text { Dist. } \\
\text { const }\end{array}$ & $\begin{array}{c}\text { Accumul- } \\
\text { ation }\end{array}$ & Sodium & Chloride & Sitrate & $\begin{array}{l}\text { is } \\
\mathrm{SO}_{+}{ }^{2}\end{array}$ & Reference \\
\hline & & & $\mathrm{m}$ & $\mathrm{km}$ & $\mathrm{mma}^{\prime}$ & $\mu$ eq $]^{1}$ & $\mu e q 1^{1}$ & $\mu e q 1^{1}$ & $\mu \mathrm{eq} 1^{\prime}$ & \\
\hline
\end{tabular}

\begin{tabular}{|c|c|c|c|c|c|c|c|c|c|c|}
\hline Dome $\mathrm{C}$ : & $74^{\circ} 42^{\prime} \mathrm{S}$ & $124^{\circ} 04^{\prime} \mathrm{E}$ & 3240 & 910 & 36 & 0.90 & 0.40 & 0.31 & 1.50 & $\begin{array}{l}\text { Legrand and Delmas. } \\
\qquad 1988 \mathrm{~b}\end{array}$ \\
\hline South Pole & $90^{\circ} \mathrm{S}$ & & 2880 & 1270 & 85 & 0.60 & 1.30 & 1.40 & 1.40 & Legrand and Delnas, 1984 \\
\hline Vostok & $78^{\circ} 28^{\prime} \mathrm{S}$ & $106^{\circ} 48^{\circ} \mathrm{E}$ & 3488 & 1300 & 22 & 1.20 & 0.50 & 0.25 & 2.60 & Legrand and others. 1988 \\
\hline 1)!0 & $66^{\prime} 42^{\prime} \mathrm{S}$ & $139^{\circ} 48^{\prime} \mathrm{F}$ & 270 & 4 & 153 & 19.00 & $22.14^{*}$ & & 0.37 & Drlmas and others, 1982 \\
\hline 1) 10 & $66^{\circ} 42^{\prime} \mathrm{S}$ & $139^{\circ} 48^{\prime} \mathrm{C}$ & 270 & 4 & 153 & & & 0.32 & & Legrand and Delmas. 1985 \\
\hline 1923 & $66^{\prime} 47^{\prime} \mathrm{S}$ & $139^{\circ} 40^{\prime} \mathrm{H}$ & 580 & 16 & 445 & 5.70 & $6.64^{*}$ & & & Boutron and Lorius, 1977 \\
\hline $\mathrm{D} 24$ & $66^{\circ} 48^{\prime} \mathrm{S}$ & $139^{\circ} 39^{\prime} \mathrm{E}$ & 500 & 17 & & 2.70 & 3.00 & 0.65 & 1.30 & Legrand and Delmas, 1985 \\
\hline 1) 33 & $66^{\circ} .5 l^{\prime} \mathrm{S}$ & $139^{\circ} 33^{\prime} \mathrm{E}$ & 730 & 26 & 380 & 5.70 & $6.64^{*}$ & & & Boutron and Lorius, 1977 \\
\hline $1 \ngtr 40$ & $66^{5} 54^{\prime} S$ & $139^{\circ} 29^{\prime} \mathrm{E}$ & 600 & 33 & & 2.70 & 3.10 & 0.15 & 0.30 & Legrand and Delmas, 1985 \\
\hline D12 & $67^{\circ} 03^{\prime} \mathrm{S}$ & $139^{\circ} 15^{\prime} \mathrm{E}$ & 100 & 53 & 520 & 2.90 & $3.38^{*}$ & & & Boutron and Lorius, 1977 \\
\hline D46 & $67^{\circ} 20^{\prime} \mathrm{S}$ & $138^{\circ} 48^{\prime} \mathrm{E}$ & 1450 & 93 & 390 & 1.00 & $1.17^{*}$ & & & Boutron and Lorius, 1977 \\
\hline $\mathrm{D} 4 \overline{7}$ & $6723 \mathrm{~S}$ & $138^{\circ} 43^{\prime} \mathrm{F}$ & 1300 & 100 & & 0.90 & 1.20 & 0.93 & 0.50 & Legrand and Delmas, 1985 \\
\hline 150 & $67^{\circ} 37^{\prime} \mathrm{S}$ & $138^{\circ} 20^{\prime} \mathrm{E}$ & 1700 & 130 & & 1.00 & 1.10 & 0.67 & 0.40 & Legrand and Delmas, 1985 \\
\hline D52 & $67^{\circ} 47^{\prime} \mathrm{S}$ & $138^{\circ} 07^{\prime} \mathrm{E}$ & 1800 & 150 & & 0.40 & 0.40 & 0.32 & 0.20 & Legrand and Delmas, 1985 \\
\hline 1355 & $680 \mathrm{I}^{\prime} \mathrm{s}$ & $137^{\circ} 47^{\prime} \mathrm{E}$ & 2028 & 180 & 70 & 0.30 & 0.40 & & 0.40 & Legrand and Delmas, 1985 \\
\hline D55 & $6801^{\prime} \mathrm{S}$ & $137^{\circ} 47^{\prime} \mathrm{F}$ & 2028 & 180 & 70 & & & 0.53 & & Legrand and Delmas, 1986 \\
\hline D57 & $68^{\circ} 1 \mathrm{l}^{\prime} \mathrm{S}$ & $137^{\prime} 33^{\prime} \mathrm{E}$ & 2050 & 200 & 410 & 1.00 & 1.10 & 0.66 & 0.40 & Legrand and Delmas, 1985 \\
\hline $\mathrm{D} 58$ & $68^{\circ} 15^{\prime} \mathrm{S}$ & $137^{\circ} 27^{\prime} \mathrm{E}$ & 2100 & 210 & & 0.60 & 0.70 & 0.50 & 0.30 & Legrand and Delmas, 1985 \\
\hline 1959 & $682 \mathrm{l}^{\prime} \mathrm{S}$ & $137^{\circ} 19^{\prime} \mathrm{E}$ & 2220 & 223 & 296 & 0.50 & $0.58^{*}$ & & & Boutron and Lorius, 1977 \\
\hline D61 & $6839^{\prime} \mathrm{S}$ & $13707^{\prime} \mathrm{E}$ & 2300 & 240 & & 0.70 & 1.00 & 1.40 & 0.80 & Legrand and Delmas, 1985 \\
\hline D64 & $68^{\circ}+\mathrm{l}^{\prime} \mathrm{S}$ & $136^{\circ} \cdot 48^{\prime} \mathrm{E}$ & 2300 & 270 & & 0.40 & 0.50 & 0.12 & 0.30 & Legrand and Delmas, 1985 \\
\hline $\mathrm{D} 66$ & $688^{\circ} 5 \mathrm{l}^{\prime} \mathrm{S}$ & $136^{\circ} 34^{\prime} \mathrm{E}$ & 2400 & 290 & & 0.20 & 0.40 & 1.10 & 0.50 & Lcgrand and Drlmas, 1985 \\
\hline $\mathrm{D} 70$ & $6909 \mathrm{~s}$ & $136^{\circ} 05^{\prime} \mathrm{F}$ & 2400 & 330 & & 0.60 & 0.80 & 0.90 & 0.40 & Legrand and Delmas, 1985 \\
\hline D72 & $69^{\circ} 20^{\prime} \mathrm{S}$ & $135^{\circ} 18^{\prime} \mathrm{E}$ & 2360 & 353 & 232 & 0.40 & $0.47^{*}$ & & & Boutron and Lorius. 1977 \\
\hline $\mathrm{D} 73$ & $69^{\circ} 24^{\prime} \mathrm{S}$ & $135^{\circ} 43^{\prime} \mathrm{E}$ & 2400 & 360 & & 0.60 & 0.80 & 1.20 & 0.80 & Lcgrand and Dclmas, 1985 \\
\hline 1$) 77$ & $69^{\circ} 44^{\prime} \mathrm{S}$ & $1.3 .510^{\prime} \mathrm{E}$ & 2400 & 400 & & 0.90 & 1.10 & 0.70 & 0.40 & Legrand and Delmas, 1985 \\
\hline D80 & $70^{\circ} 01^{\prime} \mathrm{S}$ & $134^{\circ} 43^{\prime} \mathrm{E}$ & 2130 & 430 & 237 & 0.11 & 0.70 & 0.89 & 0.70 & Legrand and Delmas, 1986 \\
\hline $\mathrm{D} 100$ & $71^{\circ} 34^{\prime} \mathrm{S}$ & $139^{\circ} 59^{\prime} \mathbf{E}$ & 2810 & 633 & 126 & 0.20 & $0.23^{*}$ & & & Boutron and Lorius, 1977 \\
\hline D) 10 & $7219^{\prime} \mathrm{S}$ & $13024^{\prime} \mathbf{E}$ & 2960 & 733 & 104 & 0.45 & $0.52^{*}$ & & & Boutron and Lorius 1977 \\
\hline D 120 & $73^{\circ} 04^{\prime} \mathrm{S}$ & $128^{\circ} 44^{\prime} \mathbf{H}$ & 3010 & 833 & 82 & 0.40 & $0.47^{*}$ & & & Boutron and Lorius, 1977 \\
\hline$\Lambda-3$ & $66^{\circ} 41^{\prime} \mathrm{s}$ & $139^{\circ} 56^{\prime} \mathbf{E}$ & 220 & 2 & 450 & 41.00 & 22.00 & & & Lorius and others, 1969 \\
\hline$\Lambda-\bar{j}$ & $6641 \% \mathrm{~S}$ & $139^{\circ} 55^{\prime} \mathbf{E}$ & 230 & 2 & 450 & 20.00 & 13.80 & & 4.50 & Lorius and others, 1969 \\
\hline Gap Prudhoe Gi & $6641^{\prime} \mathrm{S}$ & $13957^{\prime} \mathbf{E}$ & & 2 & & 26.00 & 22.00 & & 6.30 & lorius and others, 1969 \\
\hline Law Dome & $6630 \mathrm{~s}$ & $113^{\circ} 00^{\prime} \mathbf{F}$ & & 115 & & 5.80 & 6.10 & 0.48 & 0.39 & Ivey and others, 1986 \\
\hline Law Dome BHQ & $66^{\circ} 43^{\prime} \mathrm{S}$ & $112^{\circ} 50^{\prime} \mathrm{E}$ & 1375 & 130 & 650 & 9.10 & $10.60^{*}$ & & & Yun-gang and others, 1988 \\
\hline $\begin{array}{l}\text { Becthoven Peninsula } \\
\text { site } 1\end{array}$ & $7152 \mathrm{~S}$ & $\left(17430^{\prime} \mathrm{W}\right.$ & 580 & 16 & & & 40.10 & 0.36 & 1.52 & 'I'his paper \\
\hline $\begin{array}{l}\text { Beethoven Peninsula } \\
\text { site } 2\end{array}$ & $71^{\prime} 36^{\prime} \mathrm{S}$ & $073^{\circ} 00^{\prime} 11$ & 607 & 32 & & & 22.80 & 0.43 & $1 . \overline{50}$ & This paper \\
\hline Dolleman Island & $70^{-35} \mathrm{~S}$ & $060^{\circ} 35^{\prime}$ & 398 & 20 & 390 & & 11.76 & & 3.50 & Mulvaney and Peel, 1988 \\
\hline Dolleman Island & $7035^{\prime} \mathrm{S}$ & $060^{\circ} 55^{\prime} \mathrm{W}$ & 398 & 20 & 390 & & & 0.52 & & Mukaney and Wolff, 1993 \\
\hline Dyor Plateau & $70^{\circ} 39^{\prime} \mathrm{S}$ & $065^{\circ} 01^{\prime} \mathrm{W}$ & 1943 & 190 & & 1.19 & 1.47 & 0.50 & 0.86 & I'his paper \\
\hline GAP St 25 & $70^{\circ} 53^{\prime} \mathrm{S}$ & $06457^{\prime} \mathrm{W}$ & 1835 & 170 & 460 & 1.80 & 1.70 & & & Mumford and Peel, 1982 \\
\hline Gomez Nunatak & $74^{\circ} 01^{\prime} \mathrm{S}$ & $070^{\circ} 38^{\prime} \mathrm{W}$ & 1130 & 135 & 880 & & 2.35 & & 0.50 & Mulvaney and Peel, 1988 \\
\hline Gomez Nunatak & $74^{\circ} 01^{\prime} \mathrm{S}$ & $070^{\circ} 38^{\prime} \mathrm{W}$ & 1130 & 135 & 580 & & & 0.31 & & Mulvaney and Wolff, 1993 \\
\hline $\begin{array}{l}\text { James Ross Iskand, } \\
\text { Dome Dalinger }\end{array}$ & $64^{\prime \prime 3} \mathrm{~S}$ & $057^{\circ} 38^{\prime} \mathrm{W}$ & 1660 & 24 & 490 & 7.50 & $8.74^{*}$ & 0.40 & 1.90 & Aristarain and others, 1982 \\
\hline Puppis Pikes & $71^{\circ} 14^{\prime} S$ & $066^{\circ} 09^{\prime} 11^{\prime}$ & 1356 & 270 & & & 3.82 & 0.27 & 1.02 & This paper \\
\hline Rouley Corridor & $71^{\circ} 31^{\prime} \mathrm{S}$ & $067^{\circ} 17^{\prime} \mathrm{W}$ & 500 & 240 & & & 7.55 & 0.39 & 1.37 & This paper \\
\hline Siple & $7555^{\prime} \mathrm{S}$ & $084^{\circ} 15^{\prime} 11$ & 1054 & 250 & 560 & & 2.50 & 0.45 & 0.51 & $\begin{array}{l}\text { Musley-Thompson and } \\
\text { others, 1991 }\end{array}$ \\
\hline Stcphenson Nunatak & $72000^{\prime} \mathrm{S}$ & $069 \circ 07^{\prime}$ & 300 & 130 & & & 4.71 & 0.51 & 1.40 & This paper \\
\hline Temnikov Nunatak & $70^{\prime} 34^{\prime} \mathrm{S}$ & $06415 \mathrm{~W}$ & 1606 & 160 & & & 1.48 & 0.19 & 1.64 & This paper \\
\hline Mizuho & $70^{\circ} 42^{\prime} \mathrm{S}$ & $044^{\circ} \mathrm{F}$ & 2230 & 310 & & 0.65 & 1.10 & 0.90 & 0.89 & Osada and Higuchi. 1990 \\
\hline $\begin{array}{l}\text { Dronning Maud } \\
\text { Land }\end{array}$ & $75^{\circ} 00^{\prime} \mathrm{S}$ & $002^{\circ} 00^{\prime} \mathrm{E}$ & 2900 & 555 & 77 & 0.59 & 0.68 & 0.88 & 1.21 & $\begin{array}{l}\text { Personal communication } \\
\text { from L. Isaksson }\end{array}$ \\
\hline $\begin{array}{l}\text { Dronning Maud } \\
\text { Land }\end{array}$ & $71^{\circ} 48^{\prime} \mathrm{S}$ & $004000^{\prime} \mathrm{E}$ & & 200 & & 4.30 & 3.70 & & 0.90 & Gjessing, 1989 \\
\hline $\begin{array}{l}\text { Dronning Maud } \\
\text { Land }\end{array}$ & $71^{\circ} 58^{\prime} \mathrm{S}$ & $004^{\circ} 00^{\prime} \mathrm{E}$ & & 220 & & 2.90 & 2.10 & & 1.10 & Gjossing, 1989 \\
\hline $\begin{array}{l}\text { 1)romning Maud } \\
\text { Land }\end{array}$ & $70^{\circ} 04^{\prime} \mathrm{S}$ & $004^{\circ} 00^{\circ} \mathrm{E}$ & & 8 & & 124.00 & 140.00 & & -5.00 & Gjessing, 1989 \\
\hline $\begin{array}{l}\text { Dronning Maud } \\
\text { land }\end{array}$ & $70^{\circ} 00^{\prime} \mathrm{S}$ & $004^{\circ} 00^{\prime} \mathrm{E}$ & & 0 & & 360.00 & 420.00 & & -30.00 & Gjessing, 1989 \\
\hline Roi Baudouin & $70^{\circ} 26^{\prime} \mathrm{S}$ & $024^{\circ} 19^{\prime} \mathrm{E}$ & 0 & 12 & & 84.00 & 66.00 & & & Brocas and Delwiche. 1963 \\
\hline S- 122 & $7001^{\prime} \mathrm{S}$ & $04307^{\prime} \mathrm{E}$ & & 215 & & 2.30 & $2.68^{*}$ & & & $\begin{array}{l}\text { Murozumi and Nakimura. } \\
1974\end{array}$ \\
\hline$Y-135$ & $7127 \mathrm{~S}$ & $047^{\circ} 22^{r} \mathrm{E}$ & & 430 & & 0.50 & $0.58^{*}$ & & & $\begin{array}{l}\text { Murozumi and Nakamura. } \\
1974\end{array}$ \\
\hline
\end{tabular}




\begin{tabular}{|c|c|c|c|c|c|c|c|c|c|c|}
\hline Sile & Lat. & Long. & $\begin{array}{l}\text { Altitude } \\
\mathrm{m}\end{array}$ & $\begin{array}{c}\text { Dist- } \\
\text { coast } \\
\mathrm{km}\end{array}$ & $\begin{array}{l}\text { Accumul- } \\
\text { ation } \\
\operatorname{mma}\end{array}$ & $\begin{array}{l}\text { Sodium } \\
\text { Heq }^{1}\end{array}$ & $\begin{array}{l}\text { Chioride } \\
\text { ucq I }^{\prime}\end{array}$ & $\begin{array}{l}\text { Nirate } \\
\text { Heql }^{1}\end{array}$ & $\begin{array}{c}x w \\
\mathrm{SO}_{4}{ }^{2} \\
\mu e \mathrm{eq}{ }^{2}\end{array}$ & Refermae \\
\hline$Y-200$ & $71^{\circ} 46^{\prime} \mathrm{s}$ & $048^{\circ} 56^{\prime} \mathrm{E}$ & & 490 & & 0.60 & $0.70^{*}$ & & & $\begin{array}{l}\text { Murozumi and Nakamura, } \\
1974\end{array}$ \\
\hline$Y-300$ & $70^{\circ} 55^{\prime} \mathrm{s}$ & $049^{\circ} 53^{\prime} \mathrm{E}$ & & 440 & & 0.50 & $0.58^{*}$ & & & $\begin{array}{l}\text { Murozumi and Nakamura, } \\
1974\end{array}$ \\
\hline$B+55$ & $81^{\circ} 28^{\prime} \mathrm{s}$ & $060^{\circ} 36^{\prime} \mathrm{W}$ & & 530 & 92 & & 5.13 & 0.78 & $1.16(0.84)$ & Minikin and others, 1994 \\
\hline B. +56 & $81^{\circ} 36^{\prime} \mathrm{s}$ & $058^{\circ} 10^{\prime} 11$ & & 530 & 89 & & 5.44 & 1.15 & $1.25(0.92)$ & Minikin and others, 1994 \\
\hline BC90 & $76^{\circ} 59^{\prime} \mathrm{S}$ & $\left.052^{\circ} 28^{\prime} 1\right\}^{\prime}$ & & 14 & 204 & & 27.00 & 0.78 & & Graf and others, 1991 \\
\hline Berkner Island & $7936^{\prime} \mathrm{S}$ & $04537^{\prime} 11$ & 900 & 150 & 174 & & 16.08 & 0.55 & $1.75(0.77)$ & $\begin{array}{l}\text { Wagenbach and others, } \\
1994\end{array}$ \\
\hline Berkner Island & $78^{\prime} 18^{\prime} \mathrm{S}$ & $046^{\circ} 17^{\prime} 1 t^{\prime}$ & 730 & 50 & 226 & & 29.62 & 0.52 & $2.33(0.53)$ & $\begin{array}{l}\text { Wagenbach and others, } \\
1994\end{array}$ \\
\hline Coats land & $7702^{\prime} \mathrm{S}$ & $022^{\circ} 32^{\prime} 11$ & 1862 & 1.50 & 155 & & 3.00 & 0.71 & 0.50 & Mulvancy and Wolli: 1993 \\
\hline Coats Land & $77 \times 34 \mathrm{~s}$ & $025^{\circ} 22^{\prime} \mathrm{WI}$ & 1420 & 145 & 59 & 2.48 & 3.34 & & 2.16 & This paper \\
\hline D131 & $76^{\circ} 58^{\prime} \mathrm{S}$ & $054^{\circ} 42^{\prime} 11$ & & 55 & 201 & & 22.48 & 0.61 & $1.85(0.48)$ & Minikin and others, 1994 \\
\hline 1)136 & $77^{\circ} 12^{\prime} \mathrm{S}$ & $053^{\circ} 08^{\prime} 11^{\circ}$ & & 70 & 189 & & 22.34 & 0.48 & $1.89(0.53)$ & Minikin and ollers, 1994 \\
\hline $\mathrm{D}_{2} 230$ & $7722 \mathrm{~s}$ & $056^{\circ} 06^{\prime} 11$ & 49 & 120 & 199 & & 13.65 & 0.59 & $1.56(0.73)$ & Minikin and others, 1994 \\
\hline D231 & $7741^{\prime} \mathrm{S}$ & $057^{\circ} 20^{\prime} \mathrm{W}$ & 54 & 160 & 185 & & 11.57 & 0.61 & $1.23<0.52$ & Minikin and others, 1994 \\
\hline 1)23.3 & $77^{\circ} 31^{\prime} \mathrm{s}$ & $0.54^{\circ} 33^{\prime} \mathrm{W}$ & & 123 & 184 & & 18.70 & 0.71 & $1.69(0.53)$ & Minikin and others, 1994 \\
\hline D236 & $77^{\circ} 56^{\prime} \mathrm{s}$ & $055.59^{\prime} 1$ & & 171 & 166 & & 14.36 & 0.73 & $1.83(0.88$ & Minikin and others, 1994 \\
\hline $\mathrm{D} 240$ & $7752^{\prime} \mathrm{s}$ & $052^{\circ} 48^{\prime} \mathrm{W}$ & 54 & 110 & 196 & & 18.28 & 0.66 & $1.69(0.58)$ & Minikin and others, 1994 \\
\hline D330 & $78^{\circ} 02^{\prime} \mathrm{S}$ & $056^{\circ} 59^{\prime} W$ & 50 & 185 & 176 & & 10.86 & 0.58 & 1.550 .89 & Minikin and others, 1994 \\
\hline D335 & $7818 \mathrm{~s}$ & $056^{\circ} 59^{\prime} \mathrm{W}$ & 51 & 221 & 170 & & 11.09 & 0.93 & $1.67(1.00)$ & Minikin and others, 1994 \\
\hline D 336 & $78^{\circ} 43^{\prime} \mathrm{s}$ & $057^{\circ} 5 \mathrm{l}^{\prime} \mathrm{W}$ & & 272 & 156 & & 11.31 & 0.82 & $1.64(1.02)$ & Minikin and others, 1994 \\
\hline D3 10 & $78^{\circ} 36^{\prime} \mathrm{S}$ & $055^{\circ} 26^{\prime} \mathrm{W}$ & 56 & 210 & 155 & & 10.07 & 0.79 & $1.540 .93)$ & Minikin and others, 1994 \\
\hline $13+3+1$ & $78.57^{\prime} \mathrm{S}$ & $056^{\circ} 50^{\prime} W$ & 60 & 255 & 137 & & 8.94 & 0.84 & 1.210 .67 & Minikin and others, 1994 \\
\hline Filchiner Ice Sheld & $79^{\circ} 00^{\prime} \mathrm{s}$ & $05700^{\prime} \mathrm{W}$ & & 270 & 140 & & & 0.50 & & Moser and others, 1990 \\
\hline $\begin{array}{l}\text { Georg ron } \\
\text { Neumayer }\end{array}$ & $70^{\circ} 00^{\prime} \mathrm{S}$ & $008^{\circ} 00^{\prime} 11^{\prime}$ & - & 0 & 350 & & & 0.30 & & Moser and others, 1990 \\
\hline Rutford & $78^{\prime} 33^{\prime} \mathrm{S}$ & $082^{\circ} 58^{\prime} \mathrm{W}$ & 160 & 420 & & & 4.93 & 0.86 & 0.62 & This paper \\
\hline Ski Hi depot & $74^{\circ} 58^{\prime} \mathrm{S}$ & $070^{\circ} 46^{\prime} \mathrm{W}$ & 1372 & 220 & & & 2.16 & 0.94 & 0.65 & This paper \\
\hline Base camp & $8228^{\prime} \mathrm{S}$ & $166^{\circ} 00^{\prime} W$ & & 450 & & 4.30 & $5.01^{*}$ & & & $\begin{array}{l}\text { Warburton and Linkletter, } \\
1977\end{array}$ \\
\hline C-16 & $81^{\circ} 11^{\prime} \mathrm{S}$ & $170^{\circ} 29^{\prime} \mathrm{E}$ & 70 & 360 & 100 & & 4.40 & 0.60 & 0.83 & Herron: 1982 \\
\hline $\mathrm{C}-7$ & $78^{\circ} 58^{\prime} \mathrm{S}$ & $176^{\circ} 00^{\prime} \mathrm{W}^{\prime}$ & 0 & 70 & & 8.50 & $9.90^{*}$ & & & $\begin{array}{l}\text { Warburton and Linkletter, } \\
1977\end{array}$ \\
\hline$C-\bar{i}-1$ & $7830^{\prime} \mathrm{s}$ & $177000^{\prime} \mathrm{W}$ & & 0 & 130 & 26.00 & $30.29^{*}$ & & & $\begin{array}{l}\text { Herron and Langway, } \\
1979\end{array}$ \\
\hline E.-8 & $84^{\circ} 37^{\prime} \mathrm{S}$ & $169^{\circ} 42^{\prime} 1$ & & 670 & & 0.50 & $0.58^{*}$ & & & $\begin{array}{l}\text { Warburton and linkletter, } \\
1977\end{array}$ \\
\hline F-8 & $84^{\prime} 18 \mathrm{~s}$ & $172 \cdot 12^{\prime} 15$ & & 640 & & 1.50 & $1.75^{*}$ & & & $\begin{array}{l}\text { Warburton and Linkletter, } \\
1977\end{array}$ \\
\hline Gi-8 & $83^{\circ} 30^{\prime} \mathrm{S}$ & $171000^{\prime} \mathrm{W}$ & & 580 & & 1.40 & $1.63^{*}$ & & & $\begin{array}{l}\text { Warburton and Linkletter, } \\
1977\end{array}$ \\
\hline $\mathrm{H}-9$ & $83^{\circ} 11^{\prime} \mathrm{s}$ & $168^{\circ} 48^{\prime}$ & & 520 & & 1.05 & $1.22^{*}$ & & & $\begin{array}{l}\text { Warburton and Linkletter, } \\
1977\end{array}$ \\
\hline $\mathrm{I}-8$ & $82^{-} 30^{\prime} \mathrm{S}$ & $165^{\circ} 12^{\prime} \mathrm{M}$ & & 460 & & 1.95 & $2.27^{*}$ & & & $\begin{array}{l}\text { Warburton and Linkletter, } \\
1977\end{array}$ \\
\hline $\mathrm{J}-8$ & $82^{\circ} 05^{\prime} \mathrm{S}$ & $165^{\circ} 24^{\prime} \mathrm{W}$ & & 100 & & 1.40 & $1.63^{*}$ & & & $\begin{array}{l}\text { Warburton and Linkletter, } \\
1977\end{array}$ \\
\hline$J-9$ & $8222 \mathrm{~s}$ & $168^{\prime} 40^{\prime} W$ & 55 & 450 & 90 & 4.30 & $5.01^{*}$ & & & $\begin{array}{l}\text { Herron and Langway, } \\
1979\end{array}$ \\
\hline$[-7$ & $81^{c} 25^{\prime} \mathrm{S}$ & $16500^{\prime} \mathrm{W}$ & & 280 & & 2.10 & $2.45^{*}$ & & & $\begin{array}{l}\text { Warburton and Linkletter, } \\
1977\end{array}$ \\
\hline Little America V & $78=10^{\prime} \mathrm{S}$ & $162^{\circ} 13^{\prime}$ & & 0 & & 19.00 & $22.14^{*}$ & & & Langway and others, 1974 \\
\hline $.1-7$ & $80^{\prime} 56^{\prime} \mathrm{S}$ & $164 \% 06^{\prime} \mathrm{W}$ & & 230 & & 4.10 & $4.78^{*}$ & & & $\begin{array}{l}\text { Warburton and linkletter, } \\
1977\end{array}$ \\
\hline$Q-13$ & $7857^{\prime} \mathrm{S}$ & $179^{\circ} 55^{\prime} \mathrm{E}$ & 50 & 130 & 140 & & & 0.70 & 1.20 & Herron, 1982 \\
\hline RIC & $80^{\circ} 11^{\prime} \mathrm{S}$ & $16148^{\prime} \mathrm{W}$ & & 150 & & 3.80 & $4.43^{*}$ & & & $\begin{array}{l}\text { Warburton and Linkletter, } \\
1977\end{array}$ \\
\hline Rousevelt Island & $79^{\circ} 22^{\prime} \mathrm{s}$ & $161^{\circ} 40^{\prime} \mathrm{W}$ & & 130 & & 5.70 & $6.64^{*}$ & & & $\begin{array}{l}\text { Warburion and Linkletter, } \\
1977\end{array}$ \\
\hline Ross Is Windless Bight & $77^{\circ} 45^{\prime} \mathrm{S}$ & $167^{\circ} 00^{\prime} \mathrm{E}$ & & 0 & & & 12.00 & 1.00 & 2.00 & Palais and others, 1989 \\
\hline Dominion Rangc & $85^{\prime} 15^{\prime} \mathrm{S}$ & $166^{\circ} 10^{\prime} \mathrm{E}$ & 2780 & 1100 & 35 & & & 1.60 & & Lyons and others, 1990 \\
\hline N. Nictoria Land E 10 & $72^{\circ} 00^{\prime} \mathrm{s}$ & $165^{\circ} 00^{\prime} \mathrm{E}$ & 1100 & 130 & 160 & 3.60 & 4.20 & 0.60 & 1.00 & Allen and others, 1985 \\
\hline N.Victoria Land Ml & $72^{\circ} 00^{\prime} \mathrm{S}$ & $162^{\circ} 00^{\prime} \mathrm{E}$ & 2305 & 230 & 160 & 3.80 & 4.60 & 0.82 & 1.10 & Allen and others, 1985 \\
\hline South Victoria land & $7732 \mathrm{~S}$ & $160^{\circ} 35^{\prime} \mathbf{E}$ & & 100 & & & & 1.10 & & Lyons and others, 1990 \\
\hline Byrd & $79^{\circ} 59^{\prime} \mathrm{S}$ & $120^{\circ} 01^{\prime} \mathrm{W}$ & 1530 & 700 & 160 & & 1.70 & 0.65 & 0.71 & Herron, 1982 \\
\hline
\end{tabular}

"Indicates where Cl values have been calculated from $\mathrm{Na}^{+}$given in the original reference, using normal sea-salt ratios.

Figures in brackets are the $\mathrm{xs} \mathrm{SO}_{4}{ }^{2}$ concentrations for the Filchner-Ronne Ice Shelf calculated in the conventional manner using normal sea-salt ratios. 


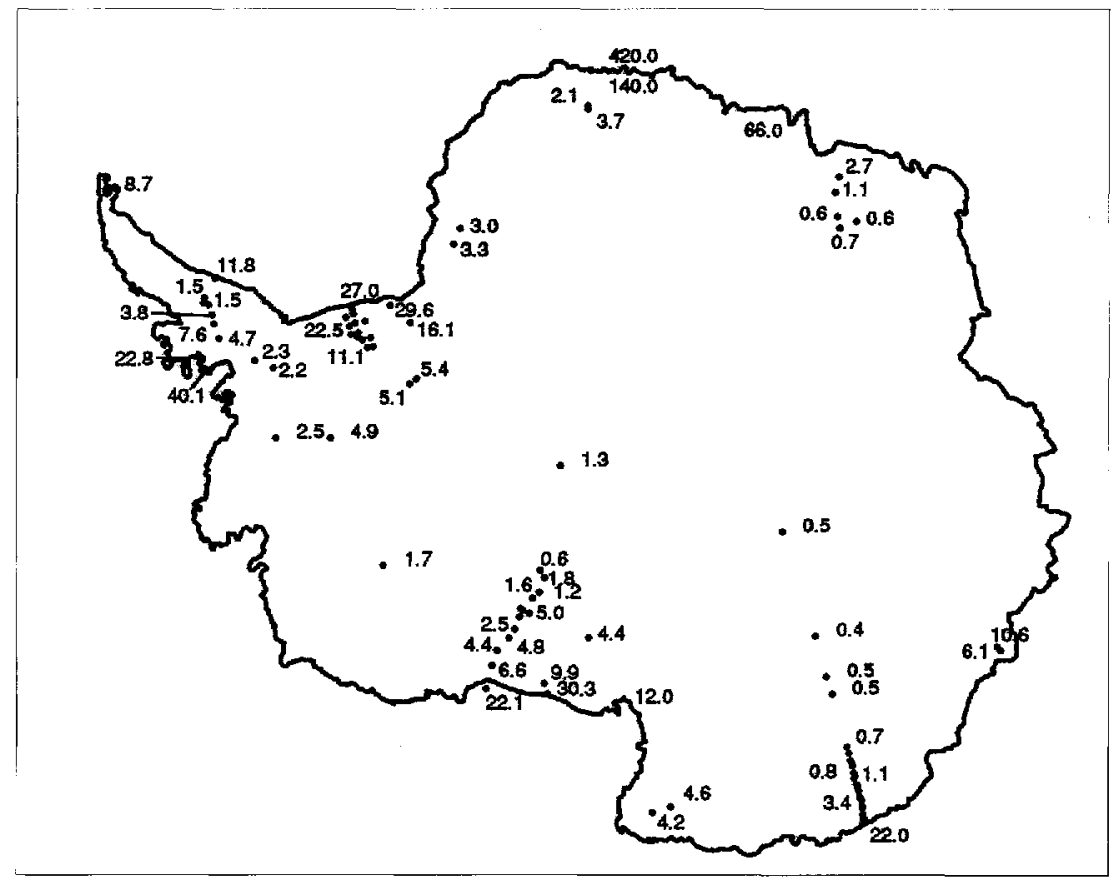

Fig. 1. The spatial distribution of $C T$ data across Antarctica is shown along wilh $C$ concentrations in $\mu$ eq $l^{1}$. In areas where there are a great number of data points, selected concentrations only have been given for clarity.

shelves, distance from the coast varies independently of altitude, so that this factor alone can be investigated.

In Figure 4, we have plotted the data against distance. The much higher $\mathrm{CI}^{-}$values near the coast on the Filchner-Ronne Ice Shelf compared to the Ross Ice Shelf are perhaps due to diflerences in width of fringing sea ice so that the true distance to a large body of open water is implied to be greater at the Ross Ice Shell). There would also be an effect if there were greater wind specds carrying sea spray to the FRIS. The rate of decline in concentration with distance will be controlled by scavenging losses during transport, which are governed by factors such as wind speed and accumulation rate.

The data from Berkner Island lie within the trend of the FRIS data. The implication is that, at least across open topography as on FRIS, altitude at least up to $1000 \mathrm{~m}$ ) has little additional effect on concentrations. However, it is also apparent that at other sites with steep coastal topography (e.g. the line of D sites and Coats Land) there is a much more rapid dccrcasc than on open ice shelves. There is no indication of higher concentrations at low-accumulation sites (Dome C, Vostok) and

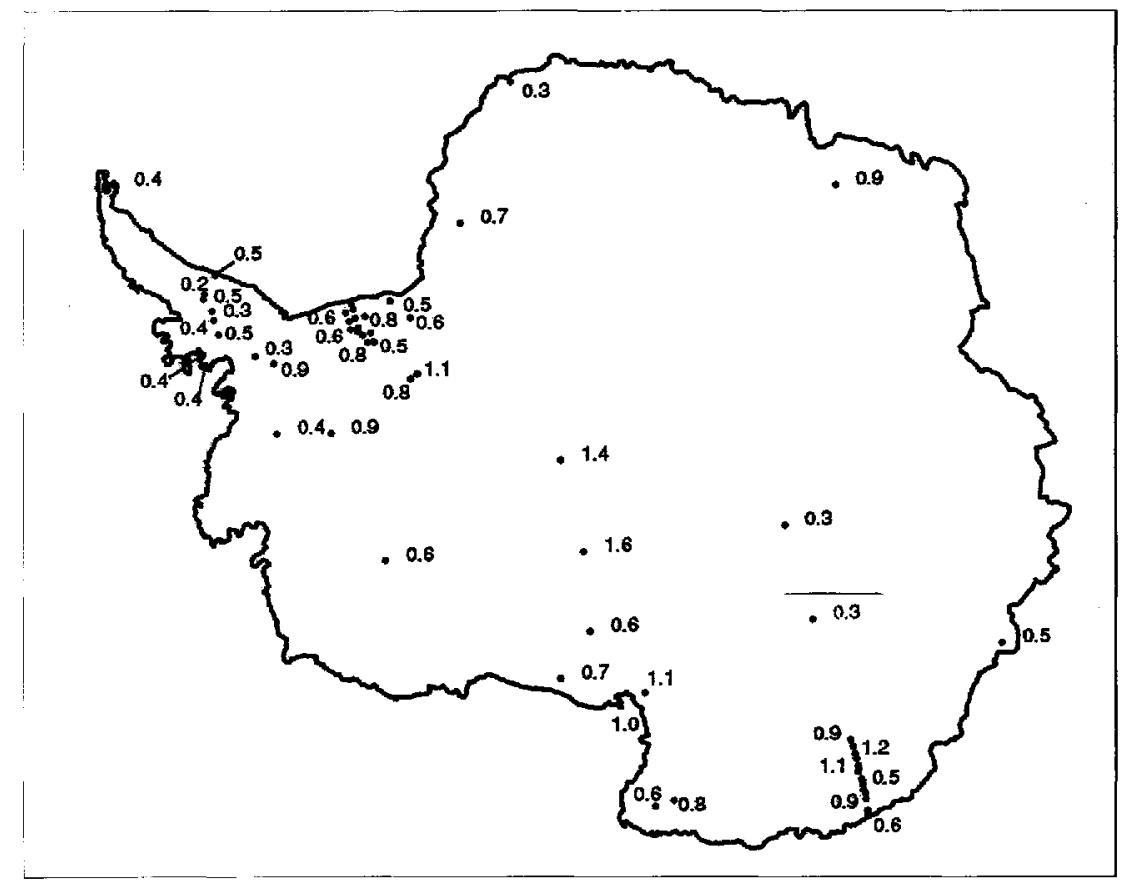

Fig. 2. The spatial distribution of $\mathrm{NO}_{3}{ }^{-}$data across Antarctica is shown along with $\mathrm{NO}_{3}{ }^{-}$concentrations in $\mu$ eql ${ }^{1}$. In areas where there are a great number of data points, selected concentrations only have been given for clarity. 


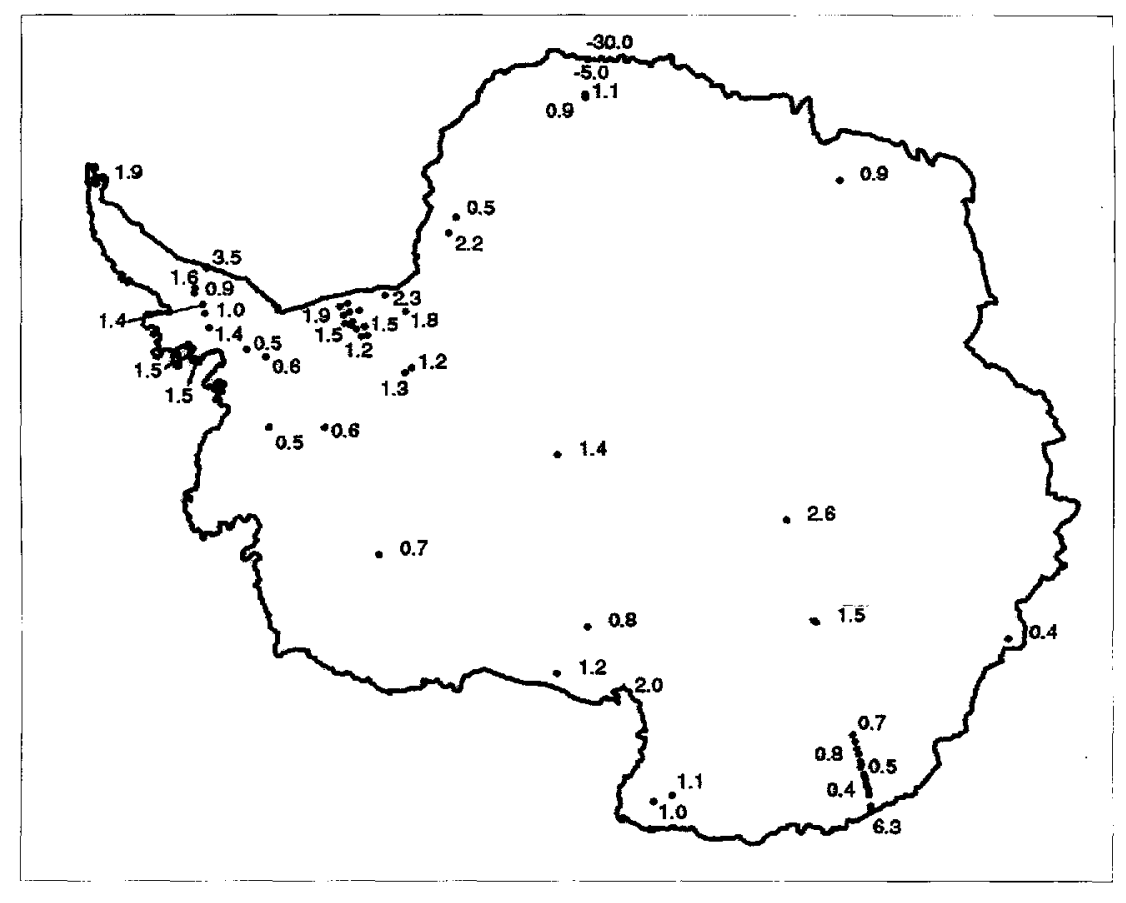

Fig. 3. The spatial distribution of $x \mathrm{SO}_{4}{ }^{2}$ data across Antarctica is shown along with $x_{5} \mathrm{SO}_{4}{ }^{2}$ concentrations in $\mu$ eq ${ }^{2}$, calculated as described in the lext. In areas where there are a great number of data points, selected concentrations only have been given for clarity.

high-altitude central eastern sites show uniformly low concentrations below $1 \mu \mathrm{eql}$ '. Finally, the ratio $\mathrm{Cl}^{-} / \mathrm{Na}^{+}$ is close to the sea-water ratio at most sites, with notable exceptions at very low-accumulation rate sites - Dome $\mathbf{C}$ and Vostok. This may imply post-depositional evaporation of $\mathrm{HCl}$, analogous to the effects suggested for $\mathrm{HNO}_{3}$ (Mayewski and Legrand, 1990; Wolff, in press).

\section{2. $\mathrm{NO}_{3}^{-}$}

The main feature of $\mathrm{NO}_{3}$ (present mainly as $\mathrm{HNO}_{3}$ ) concentrations across Antarctica is their extraordinary uniformity. The Filchner Ronne Ice Shelf data indicate that there is no effect of distance from the sea (Minikin and others, 1994). Plots of $\mathrm{NO}_{3}$ (Fig. 5a and b) against altitude and accumulation rate show that there is a tendency for $\mathrm{NO}_{3}{ }^{-}$to increase with altitude but decrease with accumulation rate. There are 1 wo outliers- Vostok

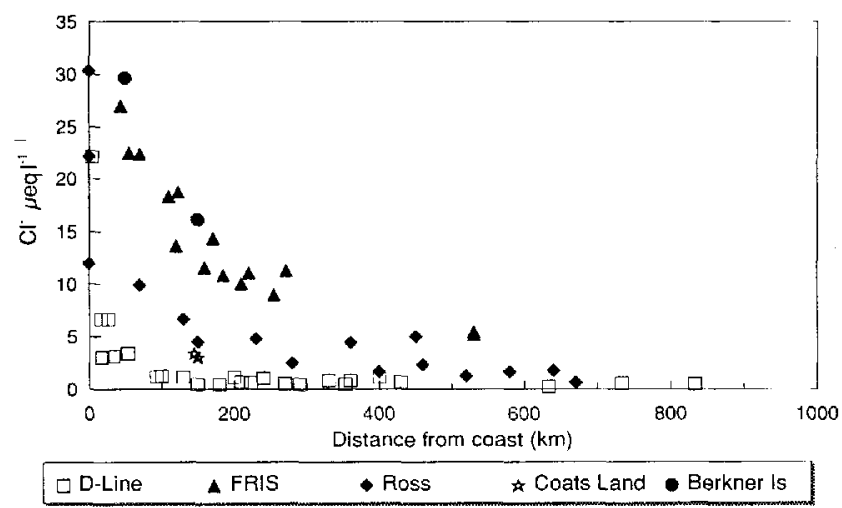

Fig. 4. The concentration of $C T$ from the French chain of $D$ sites, the Ross Sea, the Filchner Ronne Ice Shelf, including Berkner Island, and Coats Land plotted against distance from the coast. and Dome $\mathrm{C}$. Profiles of $\mathrm{NO}_{3}$ against depth from these sites show a steady decrease in concentration from the surface to approximately $1 \mathrm{~m}$ (Maycwski and Legrand,

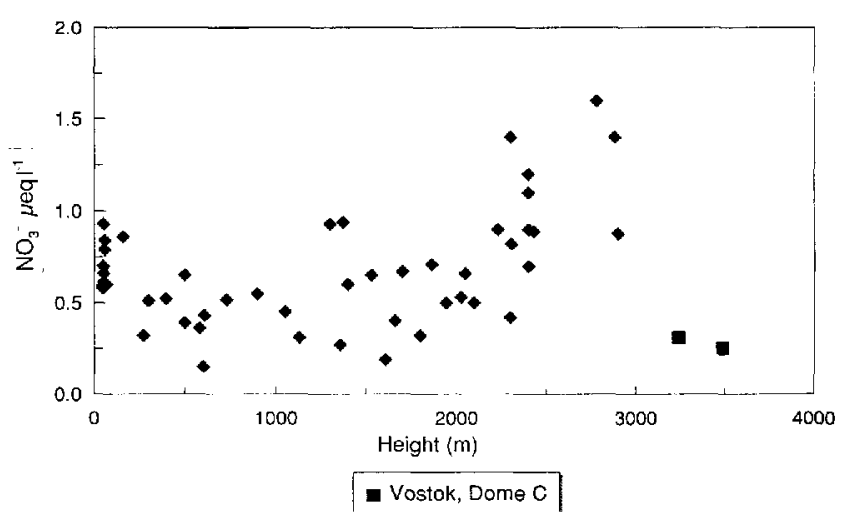

a

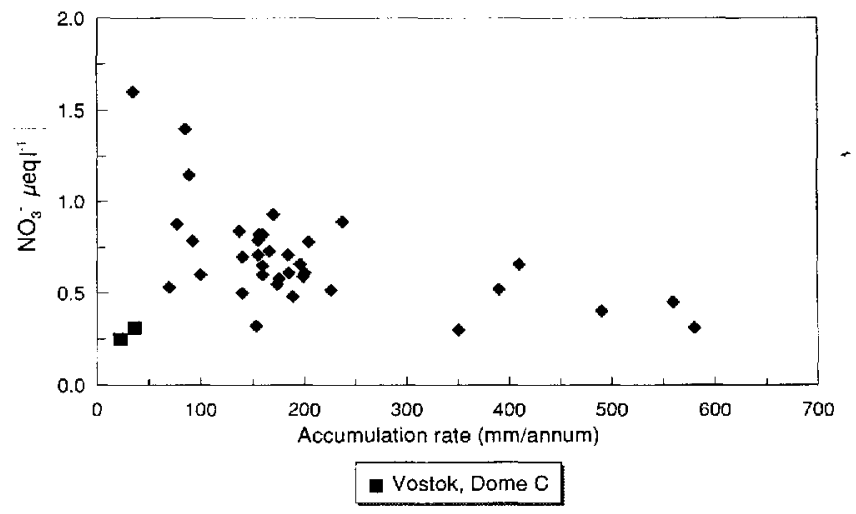

b

Fig. 5. a. $\mathrm{NO}_{3}^{-}$versus altitude; b. $\mathrm{NO}_{3}^{-}$versus accumulalion range. 
1990). This has been explained as resulting from a postdepositional loss of $\mathrm{HNO}_{3}$ from the snow, either by evaporation or perhaps photolysis, at these sites where it takes a decade or more for $1 \mathrm{~m}$ of snow to accumulate (Neubauer and Heumann, 1988; Woll, in press). For the remaining sites, $\mathrm{NO}_{3}$ appears to incrcasc with altitude, though very low-altitude sites do not fit well with this trend. An altitude dependence might imply a $\mathrm{NO}_{3}{ }^{-}$ source high in the atmosphere, perhaps from the stratosphere. The two major expected sources for $\mathrm{NO}_{3}$ (Legrand and Kirchner, 1990) are the stratosphere and tropical lightning, from which $\mathrm{NO}_{r}$ may be transported through the stratosphere. Kamiyama and others (1989) and Dahe and others (1992) both noted very high $\mathrm{NO}_{3}{ }^{-}$ concentrations in surface snow at high-altitude sites. An accumulation-rate dependence would imply a high proportion of dry deposition at contral Antarctic sites, or it may be explained by a relatively constant flux of $\mathrm{NO}_{3}$ to the surface, with dilution at higher-accumulation sitcs. It is clear that we do not yet understand fully the deposition mechanism of $\mathrm{NO}_{3}$ and further investigation is urgently required.

\section{3. $\mathrm{xSSO}_{4}{ }^{2-}$}

Data from both FRIS and the Ross Ice Shelf show xs $\mathrm{SO}_{4}{ }^{2}$ decrcasing with distance from the coast (Fig. 6), while the French line of D sites shows no similar effect. However, it should be pointed out that, when the FRIS data are recalculated using conventional sea-salt ratios, the trend in the data is more similar to that of the $\mathrm{D}$ sites. Mulvaney and others (1993) found that the concentration of $\mathrm{Cl}$ falls off more quickly with distance from the coast than does $\mathrm{xs} \mathrm{SO}_{4}{ }^{2}$. The data presented here confirm this and the explanation is likely to be a more successful scavenging mechanism for $\mathrm{Cl}^{-}$than for $\mathrm{SO}_{4}{ }^{2-}$. $\mathrm{xs} \mathrm{SO}_{4}{ }^{2-}$ appcars largely independent of both accumulation rate and altitude, though the high concentrations at the central East Antarctic sites is slightly anomalous and may indicate the effect of dry deposition. The high concentration, dominated by large summer peaks, found at Dollcman Island is believed to be due to the close proximity of the biologically produced DMS (Mulvaney and others, 1992). Similar high values for xs $\mathrm{SO}_{4}{ }^{2}$ are also found at the coastal sites of Cap Prudhoe and A-5, and we can assume that this is also due to the productivity of the nearby ocean.

\section{CONGLUSIONS}

C.] decreases with distance from the sea but the coastal topography is also important, with open ice shelves allowing more sea salt to be carried inland than in areas with coastal mountains. No single formula involving distance from the coast and altitude describes all the data, and more data are needed to define regimes where the two factors (as well as possible post-depositional losses inland) are dominant. This may lead to insights into source areas and transport strengths across the continent. More sophisticated studies will need to look at the variation between sites in different seasons, as the seasonal variability can be hugc at some sites.

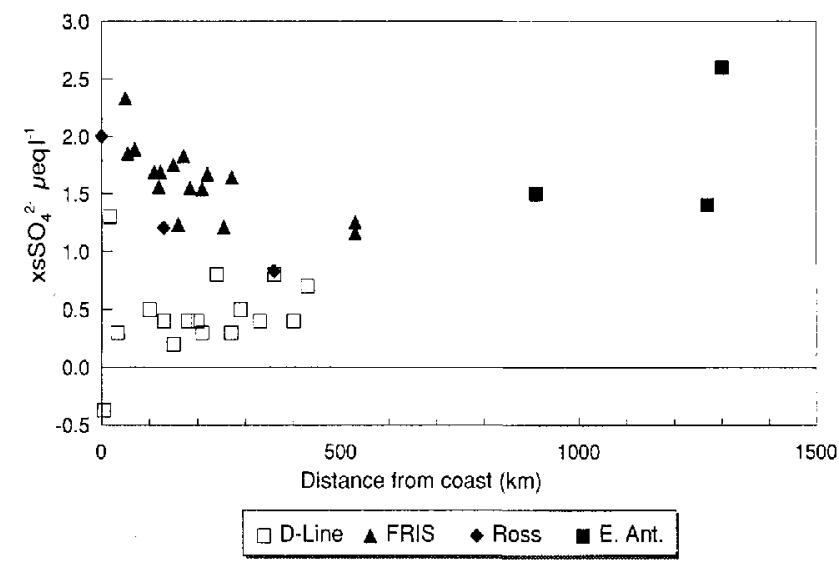

Fig. 6. The concentration of $x \mathrm{SO}_{4}$ from the French chain of D sites, the Filchner-Ronne Ice Shelf, the Ross Sea and the central East Antarctic sites against distance from the coast.

There is a remarkable uniformity in the spatial distribution of $\mathrm{NO}_{3}$ throughout Antarctica but we have noted the importance of post-depositional loss of $\mathrm{HNO}_{3}$ particularly in low-accumulation arcas. There is cridence that $\mathrm{NO}_{3}$ concentration is dependent on accumulation ratc and/or altitude. A variety of process studies is essential to understand the factors controlling $\mathrm{NO}_{3}$ deposition.

The close proximity of biologically productive areas leads to high concentrations of $\mathrm{xs} \mathrm{SO}_{4}{ }^{2}$ during the summer. Further inland, xs $\mathrm{SO}_{t}{ }^{2 .}$ decreases, though not as rapidly as $\mathrm{Cl}^{-}$. It will not be possible to explore further the significance of $x \mathrm{SO}_{4}{ }^{2}$ spatial variability until the factors controlling sea-salt fractionation are understood, so that an appropriate method of calculating $\mathrm{xs} \mathrm{SO}_{4}{ }^{2-}$ at all sites can be established.

These limited conclusions show the need for collection of additional samples along with carefully designed experiments to investigate post-depositional modification. There are large areas of Antarctica where the data are sparse and it is anticipatted that initiatives such as I'l'ASE will rectify this. The maps (Figs 1-3) point out the severe lack of data on the high polar plateau, and even around sites where multi-national deep ice-core drilling has becn proposed. Records covering at least years to decades, and several metres of snow, are needed; large numbers of very shallow cores or surface-snow samples are less useful in this respect. Understanding $\mathrm{NO}_{3}$ deposition and sea-salt fractionation at the process level is also required. With this in place, the spatial distribution of these species can offer significant insights to sources and transport that will greatly cnhance our ability to intcrpret the temporal records in ice cores.

\section{REFERENCES}

Allen, B., P. Mayewski, W.B. Lyons and M.J. Spencer. 1985. Glaciochemical studies and cstimated not mass balance for Remick Glacier area, Antarctica. Am. Glaciol, 7. 1\%6.

Aristarain, A.J., R.J. Delmds and M. Briat. 1982. Snow chemistry on James Ross Island Artaretic Peninsula J. Teophs. Res, 87 C13: $11,00111,012$

Boutron. C. and C. Lorius. 1977. Trace element content in East Antaretica snow samples. International twociation of Hodrologtal Sciences 
Publication 118 S Sumposium at Grenoble, 1975 - Isptopes and impmities in snow and ice) 164171

Brocas, J. and R. Deluiche. 1963. Cl. K, and Na concentrations in Antarctic nnow and ice. 7. Gophlys. Res., 68 13), 3999-4000.

Dahe. Q.. E.J. Zeller and G. A. M. Dreschlinfl. 1992. The distribution of nitrate content in the surface snow of the Antarctic ice sheet along the route of the 1990 International Irans-Antaretica Expedition. \% Geolders. Res, 97 A5: 6277-6284.

Delmas. R.. 11. Briat and M. Legrand. 1982. Chemistry of South Polar snow. 7. Ceophs. Res.. 87, C6 . $131+4318$.

Giovineto, M. B. and C. Bull. 1987. summary and analysis of surfare mas batance compilations for Antartica, 1960-1985. Byrd Polar Rescarch Center. Report No. 1.

Giovinetto, M. B., N. M. Waters and C. R. Bentley. 1990. Dependence of Antarctic surface mass hatance on temperature, elevation, and distunce to open ocean. 7. Geoplos. Res., 95 D4), 3517-3531.

Gjesing. Y. 1989. Excess and deficit of sulfate in polar snow. Atmos. Enith. 231 , $15.5 \cdot 160$.

Goorlach, L. and C.F. Boutron. 1992. Variations in heary metals concentrations in Antarctic snows from 1940 to 1980. F. Atmos. Chem., 14. $205-222$.

Graf. W. and 6 othess. 1991. Isolopir and chemical investigations of $10 \mathrm{~m}$ firn cores from the eastern part of the Ronne Ice Shelf, Antarctica. In Miller, H. and 11. Ocrter, eds. Filcher-Rome-1ce-Shelf-Programme, Report Va. 5. Bremerhaten. Allied Wegener Institute, 45-53

Herron. M. M. 1982. Impurity sources of $\mathrm{F}, \mathrm{Cl}, \mathrm{YO}_{3}$ and $\mathrm{SO}_{4}{ }^{2}$ in Greenland and Antarctic precipitation. ft. Geophys. Res., 87 C4t, $3052 \cdots 3060$.

Heron. M. M. and C. C. Linnway. Jr. 1979. Dating of Ross Ice Shell cores by chemical analysis. J. Geophys. Res., 2490;, 315-357.

Iver, J.P.. D. . D. Davies, V. Morgan and G. P. Avers. 1986 Nethanesulphonate in Antartic ice. Telle, 38B 15 ), 375-379.

Kamiama. K., Y. Ageta and Y. Fujii. 1989. Amospheric and clepositional environments traced from unique chemical compositions of the snow over an inland high platcals, Antarctica. F. Geophts. Res.. $94015 i, 18,515-18.519$.

Longway. C. C.. Jr. . . I Ierron and J. H. Gragin. 1971. Chemical profile of the Ross Io shelf at Lithe America V, Antarctica. F. Gtaciol. $1369,431-435$

Legrand, M. R, and R.J. Delmas. 1984. The ionic balance of Antarctic snow: a lo-sear detailed record. Almos, Eniron. 189), 1867-1874.

I.cgrand. M. and R.J. Delmas. 1985. Spatial and temporal variations of snow chemistry in 'Terre Adélic i East Antarctica). Am. Giacol, 7. 2025.

Itegand. Y.R. and R.J. Delmas. 1986. Relative contributions of troposplerie and slatompheric sources to nitrate in Antaretic snow. Tellus. 38B. 236219

I.cgrand, M. R. and R.J. Delmas. I988a. Formation of $\mathrm{IICl}$ in the Antaretic atmosphere. J. (ieophes, Res.. 93 ] 6 6, 7153-7168.

Legrand. II.R. and R.J. Delmas, 1988h. Soluble impurities in four Antaretic ice cores oxer the lats 30.000 years Am. Glacibl., 10, 116120.

Legrand. M. R. and $\$$. Kirchner. 1990. Origins and variations of nitrate in south polar precipitation. f. Geohlyw. Re's, 95 D4t, 3493-3507.

Legrand. M. and C. Saigne. 1989. Formate. acetate and methanesulphonate measurements in Antarctic ice: some geochemical implications. Atmos. Emimme, 22 5; 1011-1017.

Legrand. M. R.. C. Lorius, N. I. Barkoy and V. N. Petrov. 1988. Vostok Antarctical ice core: atmospheric chemistry changes over the last climatic crele 160.000) warsi. Atmos. Emiron., 22 2), 317-331.

Legrand. M., C. Feniet-Saigne. E. S. Saltman and C. Germain. 1992. Spatial and tempotal variations of methanesulfonic acid and non sea salt sulfate in Antartic isc. 7. Atmos. Chem., 14, 245 260.

Lorius, C. G. Baudim. J. Cintanova and R. Plater. 1969. Impuretes solubles contenues dans la glace de l'Antarctique. Tellas, 21!1!, 136-148.

Lions. W. B.. P.A. Maychski. M.J. Spencer and M. S. Twickler. 1990. Nitrate concentrations in snow from remote areas: implication for the global NO, llux. Bingendemister, 9. $211--222$.
Mavenski. P. A. and M. R. Iegrand. 1990. Recent increase in nitrate concentration of Antarctic snow. Vuture, 346/6281, 258260.

Minikin, A.. D. Wagenbach. W. Graf and J. Kipfstuhl. 1994. Spatial and seasonal sariations of the snow chemistry at the central filchner-Ronne Ice Shelf, Antaretica. Am. Ginciol., 20 isee paper in this volume:

Morgan, V. I. 1982. Antarctic ice sheet surface oxygen isotope values. F. Glaciol, 28 99 ), 315323.

Noser, K., D. Wagenhach and K. O. Minnich. 1990. Nitrate in coastal Antaretic shallow firn cores: comparison of seasonal pattern and of total flux. Abstract. Ann. Giaciol., 14, 349.

Mosley-Thompson, E., J. Dai. L. G. Thompson, P. M. Grootes, J. K. Arbogast and J.F. Paskievitch. 1991. Glaciological studies at Siple Station Antarctica: potential ice-core paleoclimatic record. 7 . Glaciol., 37:125; 11-22.

Mulvancy, R. and 1). A. Perl. 1988. Anions and cations in ice cores from Dolleman Island and the Palmer Land plateau, Antarctic Peninsula. Am. Glaciol, 10. $121 \cdot 125$

Mulvancy; R. and E.W. Wolff. 1993. Evidence for winterispring denitritication of the stratosphere in the nitrate record of Antarctic firn cores. $f$. Grophy. Res., 98 D3), 5213-5220.

Mulvancy, R., E. C. Pastcur, D. A. Pecl, E.S. Saltzman and P.-Y. Whung. 1992. The ratio of MSA to non-scat-salt sulphate in Antarctic Peninsula ice corts. Tellus, 44B, 295-303.

Mulvaney, R., G. F.J. Coulson and H. F.J. Corr. 1993. The fractionation of sea salt and acids during transport across an Antarctic ice shelf. Tellus, 45B 2 ; , 179-187.

Murnford, J. W. and D.A. Peel. 1982. Microparticles, marine salts and stable isotopes in a shallow firn core from the Antarctic Peninsula. $B r$. Antart. Surt. Bull, 56, 37-47.

Murozumi, M. and S. Nakamura. 1974. Chemical investigations of polar snow strata. In Kuroiwa. D., ed. Physical and chemical studies on ice from glacies and ice sheet. Sapporo, Hoktaido Lniversity, 6.5-75.

Neubauer, J. and K. G. Heumann. 1988. Nitrate trace determinations in snow and firn core samples of ice shelves at the Weddell Sea, Antarctica. Atmos. Enuiron., 22(3), 537-545.

Osada, $\mathrm{K}$. and $\mathrm{K}$. Higuchi, 1990. Transport rates of $\mathrm{Na}^{+}$. Cl, NO, and $\mathrm{SO}_{+}{ }^{2}$ by drifting snow at Mizuho Station, Antarctica. Proc. MPR Smp. Polar Meteorol. Gitaciol., 3, 43-50.

Palais, J. M., R. Chuan and M.J. Spcncer. 1989. Soluble and insoluble impurities in snow samples from Ross Island, Antarcticat. Ant. 7. L.S.. 245), 8991.

Savoie, D. L., J.M. Prospero, R.J. Litrsen and E.S. Saltzman. 1992. Nitrogen and sulfur species in acrosols at Mawon, Antarctica, and their relationship to ratural radionuclides. 7. Atmos. Chem., 14, 181-204.

Suttie, E. D. and E. W. Wolff. 1992. Scasonal input of heavy metals to Antarctic snow. Tellas, 44B, 351-357.

Wagenbach, D., L. Gürlach, K. Moser and K.O. Münnich. 1988. Coastal Antarctic aerosol: the seasonal pattern of its chemical composition and radionuclide conternt. Tellus, 40B $(5), 426-436$.

Wagenbach, D. and 6 athers. 1994. Reconnaissance of chemical and isotopic firn properties on top of Berkner Island, Antarctica. Ann. Glaciol., 20 sce paper in this volume:

Warburton, J.A. and G. O. Linkleter. 1977. Precipitation-forming mechanisms and the chemistry of precipitation on the Ross Ice Shelf, Antarctica. Intemational Association of IJdrological Sciences Publication 118 Symposium at Grenoble, 1975 - Tsatopes and Impurities in Snore and Ice: . 88-94.

Wolff, E. W. In press. Nitrate in polar ice. In Glabal biogeochemical cycles in pola ice. Berlin, Springer-Verlag.

Yun-gang, L., Z. Yun-hui, L. Gui-cun and X. Lun. 1988. Analysis of trace clements in the $\mathrm{BHQ}$ ice core, Law Dome, Antarctica. (Abstract.) Ann. Glaciol., 11, 219.

The accuracy of references in the text and in this list is the responsibility of the authors, to whom queries should be addressed. 